\title{
Adductor muscle contraction: reflex or response? How is the neuron irritated - via synapse or ephapse?
}

Hasan Salih Saglam¹, Dilcan Kotan², Ali Fuat Erdem³, Oztug Adsan

${ }^{1}$ Department of Urology, Faculty of Medicine, Sakarya University, Sakarya, Turkey ${ }^{2}$ Department of Neurology, Faculty of Medicine, Sakarya University, Sakarya, Turkey ${ }^{3}$ Department of Anaesthesiology, Faculty of Medicine, Sakarya University, Sakarya, Turkey

Submitted: 19 July 2016

Accepted: 24 August 2016

Arch Med Sci Civil Dis 2016; 1: e73-e74

DOI: 10.5114/amscd.2016.62040

Copyright (c) 2016 Termedia \& Banach

We have observed adductor muscle contraction during a bladder tumour resection. Here, we would like comment on an electrically induced muscle contraction explained by a hypothesis of ephaptic transmission.

An adductor muscle contraction (AMC) is a violating event that occurs during transurethral resection of a bladder tumour (TURBT), which is located on the inferolateral sides of the bladder, under epidural or spinal anaesthesia [1]. During the resection, the current of the electrocautery loop can irritate the obturator nerve $(\mathrm{ON})$, and a sudden $A M C$ can push the bladder wall to the electrocautery blade and lead to inadvertent perforation of the wall [2].

To our knowledge, an inappropriate nomenclature has been used (i.e., the term "reflex," as in obturator reflex, obturator nerve reflex, or adductor reflex) to express these undesirable contractions. For the use of a more accurate nomenclature, we aim to discuss this nomenclature in the context of AMC as well as the mechanism of inducing the contraction during TURBT in light of the literature.

A deep tendon reflex is composed of a two-neuron arc. The afferent neuron, whose cell body is in a sensory ganglion, innervates the muscle spindle. When the muscle spindle is stretched, this neuron gets excited and monosynaptically excites alpha motoneurons in the anterior horn of the spinal cord. This alpha motoneuron is the second neuron of the arc; it supplies the muscle that is transiently stretched. After a stimulus is applied to the muscle spindles, a reflex contraction results if the reflex arc is intact [3].

The absence of a reflex contraction may be due to a number of factors affecting either or both neurons. Loss of neurons and a lot of metabolic disturbance negatively affect the neurons, including diabetes, alcoholism, pernicious anaemia, some toxins, etc. [3]. General anaesthesia has been known to block the AMC when succinylcholine was administered to the patient [4]. Spinal anaesthesia has recently been shown to block the reflex arc transiently in patients with chronic complete cervical spinal cord injuries who experience spasticity of the lower extremity and positive deep tendon reflexes [5]. The deep tendon reflexes are elicited by a short, sharp blow to the tendon of an extended muscle [6]. In AMC, an electrical impulse evokes a contraction, but since there is no blow to the tendon and there is no intact reflex arc, it is not clear how this contraction happens.

Spinal or epidural anaesthesia blocks the electrical conductivity of the neurons and interneurons that are located in the spinal cord, resulting in break of the reflex arc. This is confirmed by the absence of superficial or deep tendon reflexes, such as the Babinski sign and patellar reflex,

\author{
Corresponding author: \\ Dilcan Kotan MD \\ Department of Urology \\ Faculty of Medicine \\ Sakarya University \\ Sakarya, Turkey \\ Phone: +904882143030 \\ E-mail: dilcankotan@ \\ yahoo.com
}


in patients under spinal anaesthesia. As they are located at the same lumbar nerve roots 2 to 4 as the obturator nerve, patellar reflexes have been shown to disappear after spinal anaesthesia and recover when the effects of local anaesthetics end [5]. Therefore, a muscular contraction induced by electrocautery under epidural/spinal anaesthesia may not exhibit an "excitation and reflex," since the reflex arc is broken.

Taken together, a deep tendon reflex is generated by stretching the tendon of the muscle and requires an intact reflex arc. Therefore, the contraction encountered during TURBT with a simultaneously blocked patellar reflex should be due to an electrical stimulus to the efferent fibres of the obturator nerve and referred to as a response rather than a reflex.

Another issue of interest may be how such indirect irritation of the electrical current can result in such a great unwanted contraction in the adductor muscle. The loop of the electrocautery instrument is never in contact with the obturator nerve body or its branches, but the nerve body is still evoked from the electrical current of the loop. Additionally, the contraction can be eliminated by local anaesthetics infiltration [7]. Thus the conduction of the electrical stimulus may be assumed to take place via a neural pathway. Therefore the contraction cannot be generated by any direct stimulation of the electro-cautery, which is also away from the axonal body of the obturator nerve. A possible explanation of such a muscular response to an indirect and distant stimulus may be via the hypothesis of "ephaptic transmission".

Classically, we know that electrical current can flow along a nerve to the end of the nerve fibre, and if there is a synapse, the current flow can go on via the synapse to the next neuron. This kind of electric conduction is called synaptic transmission [8]. Ephaptic transmission is conduction of a neural impulse from one nerve fibre, axon, or dendrite to another through the membranes, not along the fibres. Starting with animal models, this hypothesis was proposed to occur between mammalian cells as a transient phenomenon following acute injury of a peripheral nerve, as shown in trigeminal neuralgia and some other neurologic diseases [9].

In the hypothesis of ephaptic transmission, a large enough impulse, by generating an electric field extracellularly, can traverse the adjacent branches of other nerves that are in a position of ephapse, that is, running side by side without a direct connection. In this way, the impulse can be disseminated and can result in a strong response that is disproportionate to the initial stimulus [10]. As is well known, direct contact of the electrocautery device is never an issue with the obturator nerve, which passes inferolaterally to the bladder.
When taking the above into consideration, ephaptic transmission could be the only way for a stimulus to reach the nerve running behind the bladder wall. Ephaptic transmission has been proposed as a time- and temperature-dependent event [9]. If this experimental conclusion applies in a human model, then one could expect a beneficial application to AMC during TURBT.

According to the definition of the term "reflex," AMC during TURBT seems to be a response rather than a reflex. Therefore, the word "reflex" is a misnomer in this context. A possible explanation of such a muscular response to an indirect and distant stimulus may be via the hypothesis of "ephaptic transmission". Conduction of an electrical current from the electrocautery loop to the obturator nerve cannot happen in a synaptic way, as this is absent from this scenario; therefore, ephaptic transmission could be the only possible way that this occurs. Obviously, this hypothesis needs to be confirmed electrophysiologically in an adductor contraction during TURBT. Further studies will be necessary to identify the ephaptic transmission in animal and human models.

\section{Conflict of interest}

The authors declare no conflict of interest.

\section{References}

1. Atanassoff PG, Weiss BM, Brull SJ, et al. Compound motor action potential recording distinguishes differential onset of motor block of the obturator nerve in response to etidocaine or bupivacaine. Anesth Analg 1996; 82: 317-20.

2. Puppo P, Bertolotto F, Introini C, Germinale F, Timossi L, Naselli A. Bipolar transurethral resection in saline (TURis): outcome and complication rates after the first 1000 cases. J Endourol 2009; 23: 1145-9.

3. Walker HK. Deep tendon reflexes. In: Clinical Methods: The History, Physical, and Laboratory Examinations. $3^{\text {rd }}$ ed. Walker HK, Hall WD, Hurst JW (eds.). Butterworths, Boston 1990; 365-8.

4. Cesur M, Erdem AF, Alici HA, Yapanoglu T, Yuksek MS, Aksoy $Y$. The role of succinylcholine in the prevention of the obturator nerve reflex during transurethral resection of bladder tumors. Saudi Med J 2008; 29: 668-71.

5. Takatsuki A, Ohtsuka M. Clinical trial of a method for confirming the effects of spinal anesthesia in patients with spinal cord injury. J Anesth 2012; 26: 914-7.

6. Dick JPR. The deep tendon and the abdominal reflexes. J Neurol Neurosurg Psychiatry 2003; 74: 150-3.

7. Jo YY, Choi E, Kil HK. Comparison of the success rate of inguinal approach with classical pubic approach for obturator nerve block in patients undergoing TURB. Korean J Anesthesiol 2011; 61: 143-7.

8. Ganong WF. Review of Medical Physiology. $20^{\text {th }}$ ed. McGraw-Hill Companies, San Francisco 2001; 81.

9. Rasminsky M. Ephaptic transmission between single nerve fibres in the spinal nerve roots of dystrophic mice. J Physiol 1980; 305: 151-69.

10. Weiss SA, Faber DS. Field effects in the CNS play functional roles. Front Neural Circuits 2010; 4: 1-10. 